\title{
ANALYSIS OF TWO-CHANNEL CONTROL ACCORDING TO THE PROPORTIONAL NAVIGATION METHOD
}

\author{
L. Baranowski*
}

\begin{abstract}
In this paper the equations of the ideal constraints of the proportional navigation method have been presented, as well as the simplified equations applicable in two-channel anti-aircraft missile control. Using the elaborated mathematical model of homing the test missile some numerical experiments have been conducted, regarding the influence of the undertaken simplification in realisation of the homing method constrains on the flight dynamics and the hit accuracy of the canard configuration rocket.
\end{abstract}

Keywords: homing guidance, control systems, proportional navigation, programme constraints

\section{Introduction}

For striking an aerial target by a guided missile, it is necessary to change intentionally a direction (and sometimes a value) of a velocity vector of the missile's centre of mass. In other words, constraints should be imposed on the movement of a ballistic missile to ensure that the missile approaches a target, at the distance at which warhead striking is effective. Such constraints are called program constraints. In reality, as a result of the missile inertia and interaction of various external disturbances, the missile movement is not perfectly subordinated to the determined constraints. Thus, it is necessary to equip the missile with devices that can determine a breaching stage of these constraints and can create adequate control signals ensuring the missile movement on the required trajectory. The values, determining deviation of the missile movement from perfect constraints, are errors (deviations), that are the most frequently used as control parameters. Each system of scalar equations, describing perfect constraints, imposed on the missile movement in order to ensure the target striking, determines a homing method. A shape of the flight trajectory and necessary load factors affecting the missile depend on a chosen method and the way of its implementation. The paper presents modelling and investigations of spatial homing guidance process of the ground-to-air missile that are accomplished according to two methods of control parameters determination.

\section{Equations of constraints in a proportional navigation method}

Guidance of the missile, according to the proportional navigation method, consists in that the angular velocity of the velocity vector of the missile's mass centre $\Omega^{K}$ is proportional to the angular velocity of the missile-target line of sight (LoS) $\boldsymbol{\Omega}^{\text {los }}$, in compliance with the formula included in the paper Adler (1956)

$$
\mathbf{\Omega}^{K}=N \cdot \mathbf{\Omega}^{\text {los }}
$$

where $N$ is the coefficient of proportional navigation. In order to obtain a relationship for accelerations (or load factors), developed by the missile during perfect homing, according to the proportional navigation method, a vector multiplication of equation (1) by the velocity of the missile's mass centre $\mathbf{V}_{K}$ should be made

$$
\Omega^{K} \times \mathbf{V}_{K}=N \cdot \Omega^{l o c} \times \mathbf{V}_{K}
$$

Prof. Leszek Baranowski, PhD.: Faculty of Mechatronics \& Aerospace, Millitary University of Technology, Urbanowicza 2, 00-908, Warsaw, PL, leszek.baranowski@wat.edu.pl 
and using the known vector equation of the movement of the missile's mass centre (Liebiediew and Cziernobrowkin, 1973) in a movable reference system connected with the flight trajectory $O x_{k} y_{k} z_{k}$ (Baranowski, 2013)

$$
\mathrm{m}\left(\frac{\delta \mathbf{V}_{K}}{\mathrm{dt}}+\boldsymbol{\Omega}^{K} \times \mathbf{V}_{K}\right)=\sum \mathbf{F}_{e x t} \quad \text { or } \quad\left(\frac{\delta \mathbf{V}_{K}}{\mathrm{dt}}+\mathbf{\Omega}^{K} \times \mathbf{V}_{K}\right)=\mathbf{a}
$$

where: $\sum \mathbf{F}_{\text {ext }}$ is the sum of external forces affecting the missile, $\mathbf{a}$ is the vector of accelerations of the missile centre of mass, $\frac{\delta \mathbf{V}_{K}}{d t}$ is the local derivative of the velocity vector of the missile's mass centre.

Substituting equation (2) into equation (3), results in the expression for the accelerations vector of the missile's mass centre as a function of the angular velocity of the line of sight (LoS).

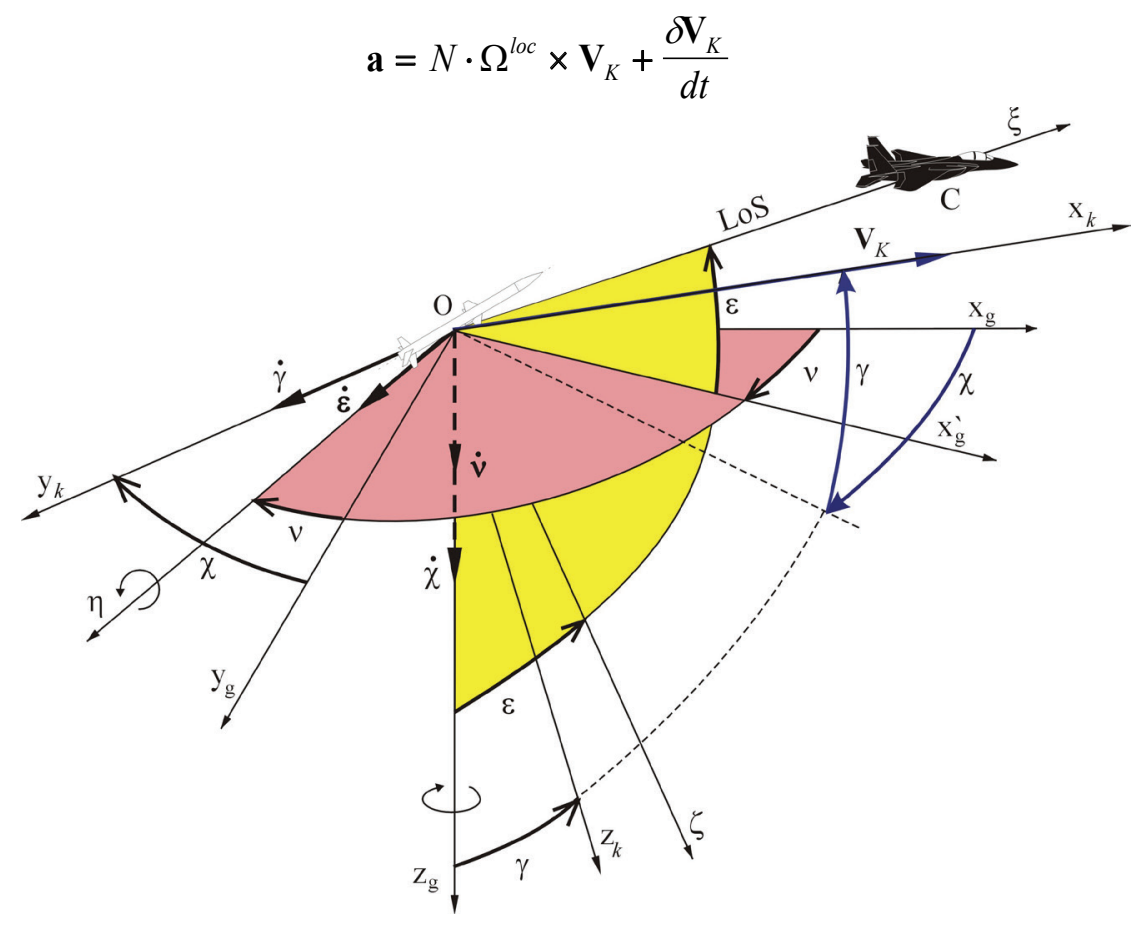

Fig. 1: Scheme illustrating the line of sight (LoS) orientation and standard coordinate systems conforming to International Standard ISO 1151.

Projecting equation (4) on the axes of the system connected with the trajectory $O x_{k} y_{k} z_{k}$ (Fig. 1), it can be obtained three equations constituting the constraints of, so-called, full control program, in compliance with the proportional navigation method

$$
a_{x_{k}}=\dot{V}_{K}, \quad a_{y_{k}}=N V_{K} \Omega_{z_{k}}^{l o c} \quad a_{z_{k}}=-N V_{K} \Omega_{y_{k}}^{l o c}
$$

Because the angular velocity $\boldsymbol{\Omega}^{\text {los }}$, according to Fig. 1, can be given in form of a sum of angular velocities of the angle of elevation $\varepsilon$ and the angle of azimuth $v$ of the line of sight

$$
\Omega^{l o c}=\dot{\boldsymbol{V}}+\dot{\boldsymbol{\varepsilon}}
$$

equations of the perfect constraints (5) take the following form

$$
a_{x_{k}}=\dot{V}_{K} \quad a_{y_{k}}=N V_{K} \cos \gamma \dot{v} \quad a_{z_{k}}=-N V_{K} \cos (\chi-v) \dot{\varepsilon}
$$

where $\gamma$ is the angle of inclination of the velocity vector of the missile's mass centre $\mathbf{V}_{K}$ and $\chi$ is the angle of azimuth of the velocity vector of the missile's mass centre $\mathbf{V}_{K}$.

For the movement control of the centre of mass of the anti-aircraft missile, there were used incomplete control programs (control of a direction and no control of a value of a velocity vector). Then, the control parameters for homing guidance, according to the proportional navigation method, could be determined from the following relations: 


$$
\begin{array}{ll}
\Delta_{1}=-N V_{K} \cos (\chi-v) \dot{\varepsilon}-a_{z_{k}} & - \text { in the elevation channel, } \\
\Delta_{2}=N V_{K} \cos \gamma \dot{v}-a_{y_{k}} & - \text { in the azimuth channel. }
\end{array}
$$

Providing the stability of a homing guidance process, for firing both from rear and front hemisphere, forces a condition for a relationship between the coefficient $N$ and the velocity of the missile, approaching the target $\dot{r}$ according to the equation

$$
N=\frac{k|\dot{r}|}{V_{K}}
$$

where $k>2$ is the constant of proportional navigation (Adler, 1956).

Using equation (10), the control parameters can be given in the following form:

$$
\begin{array}{ll}
\Delta_{1}=-k|\dot{r}| \cos (\chi-v) \dot{\varepsilon}-a_{z_{k}} & - \text { in the elevation channel, } \\
\Delta_{2}=k|\dot{r}| \cos \gamma \dot{v}-a_{y_{k}} & - \text { in the azimuth channel. }
\end{array}
$$

To reduce the costs of a unit missile, frequently complex, but required measuring devices are not applied. In practice, a simplified method of measurement and determination of control parameters is used, e.g., according to the relation:

$$
\begin{aligned}
& \Delta_{1}=-k|\dot{r}| \dot{\varepsilon}-a_{z} \\
& \text { - in the elevation channel, } \\
& \Delta_{2}=k|\dot{r}| \dot{v}-a_{y} \quad \text { - in the azimuth channel. }
\end{aligned}
$$

where $a_{z}, a_{y}$ are the normal accelerations of the missile centre of mass in the body axis system Oxyz.

\section{Results of numerical calculations and final conclusions}

An assessment of the influence of the applied simplifications in forming of the control parameters on homing process characteristics has been based upon the numerical analysis of the aero-spatial motion of the ground-to-air canard configuration test missile (Fig. 2). The test missile is a second stage of the missile discussed in the paper (Zygmunt et al, 2015) and it is stabilised in banking angle. The starting engine enables to achieve the speed of $950 \mathrm{~m} / \mathrm{s}$ in the boost phase. After dropping the starting engine the aerodynamic control system is turned on, according to the proportional navigation method with the coefficient $k=5$. Advantages and disadvantages of an alternative gas-dynamic control can be found in the paper (Głębocki, 2012).

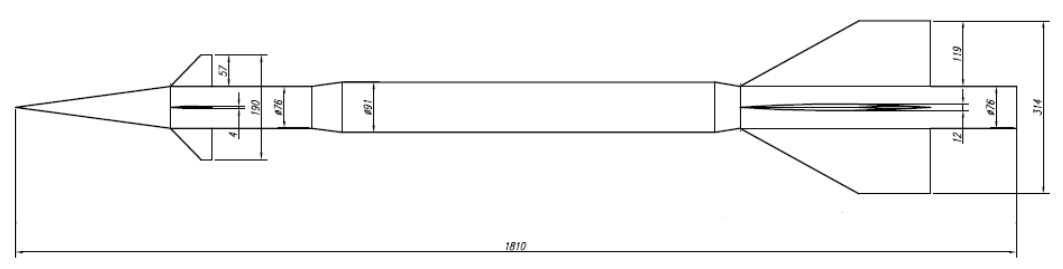

Fig. 2: Aerodynamic scheme of a test missile

For the purpose of elaboration of the mathematical model it was assumed that the missile is a rigid body with a constant mass $m=24.8 \mathrm{~kg}$, and also the control system as well as the tracking head work perfectly. The missile launcher is immobile. The complete model (without simplifications) of the tracking head can be found in (Krzysztofik et al., 2017), while the model of a vibrating launcher can be found in (Dziopa and Koruba, 2015). A mathematical model of the flight dynamics of the test missile with six degrees of freedom has been derived in a movable reference system connected with the flight trajectory $O x_{k} y_{k} z_{k}$, using the methodology described in the paper (Baranowski and Gacek, 1998). The operation of two channel control system has been described with the following equations, constituting a simplified form of control law

$$
\delta_{e}=k_{\text {amp }} \cdot \Delta_{1} \quad, \quad \delta_{a}=k_{\text {amp }} \cdot \Delta_{2}
$$

where $k_{\text {amp }}=0,0014$ - amplification coefficient, $\delta_{e}$ and $\delta_{a}$ are the rudder deflection angles in elevation and azimuth channel, respectively, taking into account the maximal possible deflection equal to 12 degrees. The full form of control laws can be found in (Ladyżyńska-Kozdraś, 2009). 
For the purpose of analysis, a series of computer simulations has been made. These simulations have been carried out for missile homing in various atmospheric conditions, during the firing from the front and the rear hemisphere to "fast" and "slow" targets, manoeuvring and not manoeuvring ones, for two variants of determination of the control parameters: according the complete relationships $(11,12)$ and according to the simplified relationships $(13,14)$.

The example calculation results, which enable to define the effect of forming the control parameters on the dynamics and the homing guidance accuracy, have been presented in comparison graphs in Fig. 3. The presented changeability of the flight parameters refer to firing from the front hemisphere to the target $\left(V_{t}=300 \mathrm{~m} / \mathrm{s}\right)$ which starts the manoeuvre with the load factor equal to $-4 \mathrm{~g}$ at $5 \mathrm{sec}$ before the estimated contact with the missile, in normal atmospheric conditions. The blue line denotes the homing process carried out according to the complete relationships of the control parameters, while the red line denotes the simplified relationships. Both, the presented graphs, and the results of the conducted research works indicate insignificant influence of the applied simplifications on the process and accuracy of homing of the tested missile (differences in accuracy less than 1 meter). They confirm the correctness of the used solutions for canard configuration missiles. In case of usage the missiles of different configuration, additional simulations need to be made.
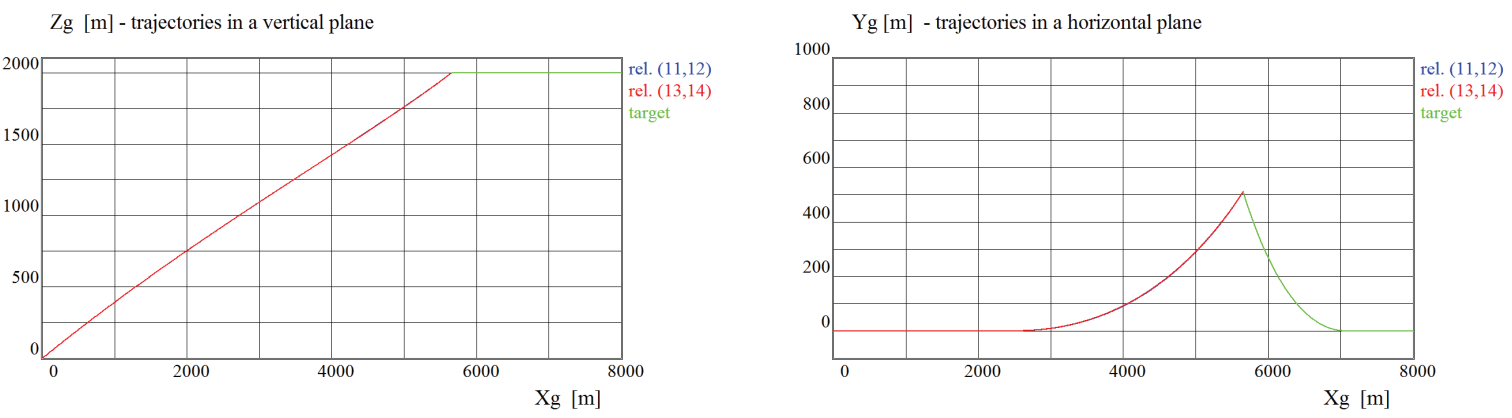

rudder deflection angles in elevation channel $[\mathrm{deg}]$
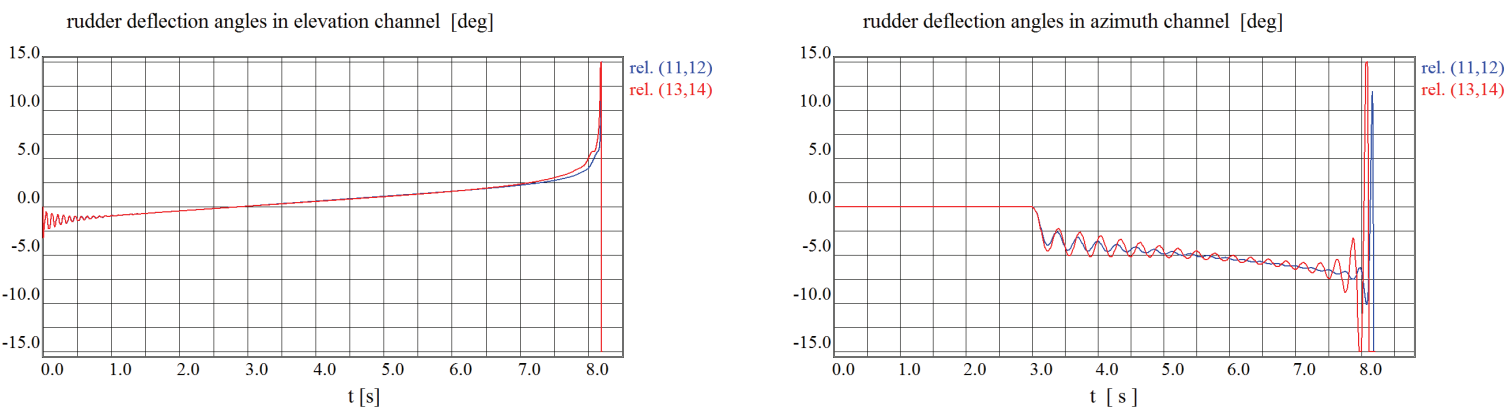

Fig. 3: Influence of a method of control parameters determination on the missile's flight parameters.

\section{References}

Adler, P. F. (1956) Missile Guidance by Three-Dimensional Proportional Navigation. Journal of Applied Physics, 27, 5, pp. 500-507.

Baranowski, L. and Gacek, J. (1998) Modeling of the self-guiding process of a medium-range anti-aircraft missile in variable atmospheric conditions, in: Proc. 8th Int. Conf. Mechanics in Aviation (eds. Maryniak, J.), PTMTS, Warsaw, pp. 15-16, (in Polish).

Baranowski, L. (2013) Effect of the mathematical model and integration step on the accuracy of the results of computation of artillery projectile flight parameters, Bull. Pol. Ac.: Tech., 61, 2, pp. 475-484.

Dziopa, Z. and Koruba, Z. (2015) The impact of launcher turret vibrations control on the rocket launch. Bull. Pol. Ac.: Tech., 63, 3, pp.717-728.

Głębocki, R. (2012) Guidance impulse algorithms for air bomb control, Bull. Pol. Ac.: Tech., 60, 4, pp. 825-833.

Krzysztofik, I., Takosoglu, J. and Koruba, Z. (2017) Selected methods of control of the scanning and tracking gyroscope system mounted on a combat vehicle. Annual Reviews in Control, 44, pp. 173-182.

Liebiediew, A. A. and Cziernobrowkin, L. C. (1973) Flight Dynamics. Mashinostroenie, Moscow, (in Russian).

Ładyżyńska-Kozdraś, E. (2009) The control laws having a form of kinematic relations between deviations in the automatic control of a flying object. Journal of Theoretical and Applied Mechanics, 47, 2, pp. 363-381.

Zygmunt, B., Motyl, K., Machowski, B., Makowski, M., Olejniczak, E. and Rasztabiga, T. (2015) Theoretical and experimental research of supersonic missile ballistics. Bull. Pol. Ac.: Tech., 63, 2, pp. 229-233. 